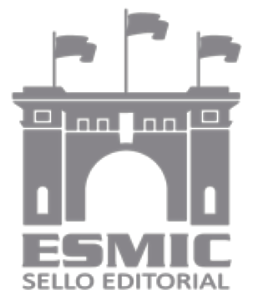

Revista Científica General José María Córdova

ISSN 1900-6586 (impreso), 2500-7645 (en línea)

Volumen 16, Número 21, enero-marzo 2018, pp. 17-31

http://dx.doi.org/10.21830/19006586.299

Citación: Hernández Méndez, J. A. (2018, enero-marzo). Amenazas nucleares, biológicas y químicas, una estrategia de manejo. Rev. Cient. Gen. José María Córdova, 16 (21), 17-31. DOI: http://dx.doi.org/10.21830/19006586.299

\title{
Amenazas nucleares, biológicas y químicas, una estrategia de manejo
}

Sección: Estudios MiLitares

Artículo de investigación científica y tecnológica

John Aldrin Hernández Méndez

Escuela de Ingenieros Militares, Bogotá, D. C., Colombia

Nuclear, biologic or chemical threats: A management strategy

Ameaças nucleares, biológicas ou químicas: uma estratégia de manejo

Des menaces nucléaires, biologiques ou chimiques: une stratégie de gestion

Recibido: 3 de agosto de 2017 • Aceptado: 28 de octubre de 2017

* https://orcid.org/0000-0003-0066-2234 - Contacto: john.hernandez@buzonejercito.mil.co 
Resumen. Esta investigación revisa la importancia que tiene elaborar un manual del Ejército colombiano para controlar el riesgo y las amenazas nucleares, biológicas o químicas (NBQ). Se considera que existe una relación entre la existencia de una unidad organizada para el manejo de incidentes en el Ejército y las posibilidades de control del uso de sustancias NBQ como armas potenciales por parte de organizaciones irregulares. La primera parte del documento hace un sucinto recuento histórico del uso de sustancias NBQ en el marco del conflicto armado interno. La segunda parte analiza brevemente las infracciones al Derecho Internacional Humanitario derivadas de dichas actividades. Finalmente, la investigación arroja luces sobre los elementos indispensables que se deben tener en cuenta en el proceso de construcción de un manual del Ejército colombiano para el control de riesgo y amenazas NBQ.

Palabras clave: amenazas nucleares biológicas o químicas; conflicto armado; manual.

\begin{abstract}
This study reviews the importance of creating a Colombian Military manual to control nuclear, biologic or chemical (NBC) risk and threats. It is believed that there is a relationship between the existence of an organized incident management unit in the Army and the possibility of controlling the use of NBC substances as potential weapons by irregular organizations. The first part of the document offers a brief history of the use of NBC substances within the frame of the internal armed conflict. The second part, briefly analyzes the infractions to International Humanitarian Rights resulting from such activities. Lastly, this study sheds light on some of the essential elements to consider during the construction process of a Colombian Military manual to control NBC risk and threats.
\end{abstract}

Keywords: armed conflict; manual; nuclear threats, biologic or chemical.

Resumo. Esta investigação revisa a importância de elaborar um manual do Exército colombiano para controlar o risco e as ameaças nucleares, biológicas ou químicas (NBQ). Acredita-se que existe uma relação entre a existência de uma unidade organizada para o manejo de incidentes no Exército e as possibilidades de controlo do uso de substâncias NBQ como armas potenciais por parte de organizaçóes irregulares. A primeira parte do documento faz um breve reconto histórico do uso de substâncias NBQ no marco do conflito armado interno. A segunda parte analisa brevemente as infracçôes ao Direito Internacional Humanitário derivadas de tais atividades. Finalmente, a investigação arroja luzes sobre os elementos indispensáveis a ter em conta no processo de construçáo de um manual do Exército colombiano para o controlo do risco e ameaças NBQ.

Palavras-chave: ameaças nucleares, biológicas ou químicas; conflito armado; manual.

Résumé. Cette recherche évalue l'importance d'élaborer un manuel de l'Armée colombienne pour contrôler le risque et les menaces nucléaires, biologiques ou chimiques (NBC). On estime qu'il y a un lien entre l'existence d'une unité organisée pour la gestion d'incidents au sein de l'Armée et les possibilités de contrôle de l'utilisation de substances NBC comme des armes potentielles par des organisations irrégulières. Dans la première partie du document, on fait un bref historique de l'utilisation de substances NBC dans le cadre du conflit armé interne. Dans la deuxième partie, on fait une brève analyse des infractions au droit international humanitaire découlant de ces activités. Enfin, cette recherche met en lumière les éléments indispensables à prendre en considération dans le processus d'élaboration d'un manuel de l'Armée colombienne pour le contrôle du risque et des menaces NBC.

Mots-clés: conflit armé ; menaces nucléaires, biologiques ou chimiques ; manuel. 


\section{El uso de sustancias nucleares, biológicas y químicas en el conflicto colombiano}

En el marco del escalonamiento del conflicto, paralelo a las conversaciones de paz del Caguán suscitadas en el periodo 1998-2002, diversos analistas militares e instituciones públicas dieron cuenta de un aumento permanente de los medios y una diversificación de los métodos con los cuales las Fuerzas Armadas Revolucionarias de Colombia (FARC) pretendían hacerse a su objetivo estratégico: la toma del poder.

Teniendo presente este objetivo, la organización irregular realizó distintas actividades, entre las cuales se incluyeron acciones para afectar el desarrollo de las negociaciones de paz mencionadas: atentados contra la población civil (presionando políticamente a la contraparte negociadora), ataques a la Fuerza Pública (presionando militarmente al gobierno), citas incumplidas, congelamiento de los diálogos y, en general, interrupciones constantes del proceso.

Este fenómeno explica por qué las FARC finalizaron el año 1998 con una de las acciones militares más arriesgadas en la historia del conflicto armado colombiano. Por primera vez en este proceso bélico, el primero de noviembre de ese año el grupo guerrillero intentó tomarse la capital de un departamento del país. Alrededor de 1.500 guerrilleros atacaron la capital del departamento del Vaupés, principalmente la estación de policía del municipio, en donde había 120 policías. Aunque el ataque fue contenido por la Fuerza Pública, que obligó a los miembros de la organización a retirarse, las FARC secuestraron a más de cincuenta policías. Tal como lo expone Camilo Echandía (1997; 2011), el grupo insurgente emplazó la toma de Mitú dentro de una estrategia general de incremento de las acciones armadas (figura 1 ).

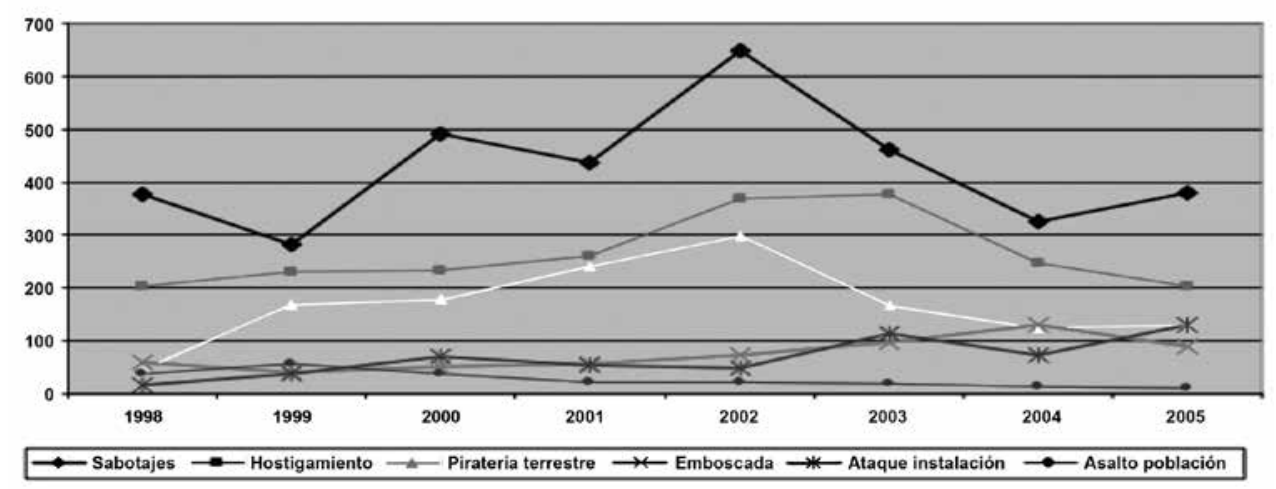

Figura 1. Evolución de las acciones más recurrentes en los grupos irregulares.

Fuente: Observatorio del Programa Presidencial de Derechos Humanos y Derecho Internacional Humanitario. 
Una de las líneas estratégicas de la guerrilla fue incluir métodos y medios prohibidos por el Derecho Internacional Humanitario (DIH), en particular, el uso de agentes químicos y biológicos como armas para lograr que los miembros de las Fuerzas Militares se replegaran o quedaran inactivos. Los ataques de las FARC se concentraron en el sur del país, principalmente en departamentos amazónicos y territorios del piedemonte amazónico, en donde la organización pretendió debilitar la presencia estatal en los escenarios locales, con el fin de acumular recursos para lanzar su ofensiva general sobre Bogotá, para lo cual acudió a medios que no había explorado hasta ese entonces en el curso del conflicto armado. Al respecto, Echandía (2011) asevera que

[e]ntre los cambios más significativos registrados en la dinámica de la confrontación, se destaca también la manifiesta prioridad que las Farc le dieron, mientras estuvo vigente la Zona de Distención, a los ataques a las poblaciones para destruir los puestos de policía y debilitar la presencia estatal en los municipios donde esta guerrilla buscó ampliar su influencia. (p. 155)

En marzo de 2003, el General Jorge Mora denunció ante la opinión pública el uso que la guerrilla estaba haciendo de cilindros bomba con mezclas de pegantes industriales y gasolina, así como la implementación de proyectiles impregnados con sustancias letales, los cuales eran empleados de forma recurrente y sistemática por algunos frentes de la guerrilla para atacar a las instituciones públicas y a la población civil ("Las FARC y el ELN usan armas químicas", 2003).

El uso de este tipo de artefactos evidenció que esta amenaza existe en Colombia y que forma parte de los medios letales con que cuentan los actores irregulares, con lo cual ponen en especial situación de vulnerabilidad a los miembros de las Fuerzas Militares, quienes en desarrollo de las operaciones militares han debido adaptar el campo táctico y estratégico.

Uno de los efectos perjudiciales de estas armas es que resulta difícil prever su uso en cualquier enfrentamiento, ya que pueden ser diseminadas en un área cercana a un objetivo militar mediante sistemas de armamentos que no llaman la atención. Debido a esta característica, constituyen un medio para atacar tropas que se encuentran en fortificaciones o poblaciones, que se vuelven un blanco fácil de afectar.

El uso más intensivo de sustancias químicas o biológicas se hace con artefactos explosivos improvisados (en adelante, AEI), entre los cuales, la mina antipersonal es el medio más recurrente. Asimismo, es frecuente que en las minas antipersonales se empleen sustancias químicas para propiciar el sistema de iniciación, el cual incluye un explosivo (anfo, relaca, pentolita, R1, pólvora negra, entre otros) que, al estallar, produce en el accionante (civil o militar) una grave afectación — generalmente la mutilación de algún miembro- .

Pero las minas, pese a su enorme impacto, no han sido los únicos artefactos en que las FARC usaron - y usan aún — agentes químicos y biológicos como armas. En 
algunos casos, la organización ha incluido en la espoleta, el detonador o el cuerpo mismo de las granadas de 215 y 160 milímetros — de construcción artesanal— sustancias químicas y biológicas destinadas a inhibir al adversario de forma expedita. Por ejemplo, en septiembre de 2001, en el corregimiento de San Adolfo, municipio de Acevedo, en el departamento del Huila, se usó cianuro en artefactos explosivos para atacar la estación de policía, lo cual configura una infracción grave al DIH. Los cuatro agentes de policía que defendían la estación murieron después de inhalar el gas, debido a que les causó ruptura de los tejidos pulmonares y un edema que paralizó su sistema respiratorio. En esa ocasión, el Instituto de Medicina Legal dictaminó que la muerte de los uniformados se produjo por "neumonitis química".

\section{El uso de sustancias nucleares, biológicas y químicas como infracción al Derecho Internacional Humanitario}

En este apartado se analizará si el uso de agentes químicos y biológicos constituye una infracción al DIH; en este caso en particular, de acuerdo con los requisitos, clasificación y tipificación del delito estipulado en el artículo 8 del Estatuto de Roma, titulado "Crímenes de guerra". Con el fin de desarrollar este punto, se trae a colación los extractos más importantes y relevantes del texto Ataques contra personas y bienes civiles y ataques desproporcionados, segundo capítulo — sobre elementos contextuales de los crímenes de guerra-, de Héctor Olásolo Alonso (2007), en el cual se establece que

[1] os elementos contextuales de los crímenes de guerra se encuentran recogidos en el artículo 8 del ER [Estatuto de Roma]. Los elementos contextuales que forman parte del tipo objetivo de los crímenes de guerra y que por tanto deben concurrir para que una determinada conducta tenga dicha condición, son denominados normalmente elementos contextuales de carácter material, para así distinguirlos de los llamados elementos contextuales de carácter jurisdiccional, que son los que se exigen para que la CPI [Corte Penal Internacional] pueda tener competencia (son aquellos relativos al umbral de gravedad necesario para que la Corte pueda ejercitar su jurisdicción material). (Olásolo, 2007, p. 152)

Uno de los aspectos más importantes para considerar que se ha cometido un crimen de guerra es que no basta con que la conducta típica se produzca en el marco geográfico-temporal de un conflicto armado, sino que también es necesario que esta se encuentre vinculada de alguna manera con dicho conflicto armado. Por consiguiente, si no existe relación entre la conducta típica y el conflicto armado en cuyo seno se produce, no se puede hablar de crimen de guerra, sino de delito ordinario cometido en tiempo de guerra.

Otro elemento contextual de carácter material es la condición de protegido que debe tener, conforme al DIH, el sujeto u objeto pasivo sobre el cual recae la conducta típica. Así, si la acción u omisión descrita en el tipo penal recae sobre un sujeto o un bien que no se encuentra protegido por el DIH, no cabe hablar de infracción o crimen de guerra. 
Los crímenes de guerra previstos en el Estatuto de Roma se pueden dividir en tres grupos, en atención a la naturaleza de las personas o bienes sobre los cuales deben recaer: en primer lugar, se encuentran aquellos recogidos en el artículo 8(2) (a) ER, que solo pueden ser cometidos contra personas o bienes protegidos por los Convenios de Ginebra de 1949. A grandes rasgos, estos convenios ofrecen protección a las siguientes categorías de personas: $i$ ) los enfermos, heridos, náufragos, personal religioso y personal sanitario de las fuerzas armadas que hayan caído en poder del enemigo; ii) los prisioneros de guerra que hayan caído en poder del enemigo y iii) la población civil y las personas civiles que se encuentren en poder de una parte contendiente o de una potencia ocupante de la que no son nacionales o a la que no pertenecen - excepto en el caso de nacionales de Estados neutrales o cobeligerantes con representación diplomática en el Estado en cuyo poder se encuentran-.

De esta manera, la protección ofrecida por los Convenios de Ginebra no se extiende a las personas en las zonas de combate que no hayan caído todavía en poder de la parte atacante, de ahí que no afecte, en un principio, el desarrollo de las operaciones militares en situaciones de combate.

Los bienes protegidos por los Convenios de Ginebra se pueden dividir en dos categorías principales, de acuerdo con los tipos penales recogidos en el artículo 8(2)(a) del Estatuto de Roma: $i$ ) las unidades, establecimientos y transportes sanitarios, ya sean móviles o fijos, ya tengan naturaleza civil o militar, así como los bienes propiedad de sociedades de ayuda humanitaria — como el Comité Internacional de la Cruz Roja (CICR)—y ii) cualesquiera otros bienes públicos o privados que se encuentren en zona de ocupación.

Distinto es el caso del segundo subgrupo de crímenes de guerra — recogidos en el artículo 8(2)(c) del Estatuto de Roma-, que son cometidos contra aquellas personas "que no participen directamente en las hostilidades, incluidos los miembros de las fuerzas armadas que hayan depuesto las armas y las personas puestas fuera de combate por enfermedad, herida, detención o por cualquier otra causa". Como el Estatuto de Roma puntualiza, es necesario que se trate de personas que "hayan estado fuera de combate o hayan sido personas civiles o miembros del personal sanitario o religioso que no tomaban parte activa en las hostilidades".

En el tercer y último grupo de crímenes de guerra se encuentran aquellos recogidos en el artículo (8)(2)(b) y (e) del Estatuto de Roma, que solo incluyen a los cometidos contra las personas y bienes protegidos por la norma específica del DIH cuya infracción grave tipifican. En este grupo se encuentran los tres tipos penales objeto de este artículo, que se refieren solo a los cometidos contra aquellas personas u objetos que no tengan la condición de objetivo militar.

Como evidencia de que, desafortunadamente, en el conflicto colombiano se han presentado estos crímenes, se trae a colación los hechos que ocurrieron en el municipio de Acevedo (Huila) en septiembre de 2011. En esa ocasión, integrantes del Frente 61 de las FARC atacaron una estación de policía usando armas químicas y causaron la muerte por 
parálisis respiratoria de los policías que cuidaban la instalación. Este ataque constituye una de las primeras acciones unilaterales asociadas a este tipo de armamento en el conflicto colombiano. Después de usar el gas, el grupo sembró la zona con minas, lo cual impidió la entrada de personal civil y militar para atender a los policías heridos ${ }^{1}$.

En este caso concreto, es posible plantear la comisión de un crimen de guerra, de acuerdo con los términos que se detallan a continuación. Por una parte, el protocolo adicional a los Convenios de Ginebra del 12 de agosto de $1949^{2}$ recuerda un ámbito de aplicación material relacionado con la existencia y desarrollo de un conflicto armado en el territorio de jurisdicción de una Alta parte Contratante y por fenómenos distintos a las tensiones internas y de disturbios interiores, tales como los motines, los actos esporádicos y aislados de violencia y otros actos análogos, que no son conflictos armados (artículo 1).

Las FARC no solo usaron constantemente explosivos improvisados, sino que además recurrieron a la práctica ilegal de emplear armas químicas en medio de bienes y espacios de naturaleza civil, es decir, espacios que en principio no constituyen objetivos militares definidos. Aunque el Estatuto de Roma no define taxativamente cuáles son los bienes civiles en el contexto de un conflicto armado no internacional —y por ende pareciese que no es muy claro el panorama de su protección—, Olásolo (2007) sostiene que estos se pueden definir de forma negativa con base en el artículo 52 del Protocolo Adicional I, que define los bienes que son objetivos militares.

Para realizar este análisis es necesario entender la naturaleza, ubicación, finalidad o uso del bien. En el caso de la estación de policía, se entiende que es un bien de naturaleza civil —ubicado en el área rural (corregimiento de San Adolfo) del municipio de Acevedo (Huila) - adyacente a otros bienes civiles, cuya finalidad es preservar la seguridad y la convivencia en este corregimiento del país. Por esta razón, se puede afirmar que el ataque aumenta la vulnerabilidad que tienen algunas regiones cercanas al municipio de sufrir afectaciones en la provisión de servicios de seguridad y convivencia.

Por otra parte, y continuando con la definición negativa planteada por Olásolo (2007), otro argumento de que este ataque constituye un crimen de guerra se encuentra en el artículo 52(2) del Protocolo Adicional a los Convenios de Ginebra, en el cual se define explícitamente que en un conflicto armado los objetivos militares se limitan a aquellos bienes que, "por su naturaleza, ubicación, finalidad o utilización, contribuyan eficazmente a la acción militar o cuya destrucción total o parcial, captura o neutralización ofrezca en las circunstancias del caso una ventaja militar definida”. En el caso del ataque a la estación de policía de San Adolfo, no es claro de qué forma el sabotaje a esta pequeña

1 Esta incursión guerrillera forma parte de la estrategia que definió el jefe militar del Frente 61, en la cual se contemplaba el uso de artefactos explosivos improvisados y no discriminatorios de la población civil para atentar contra objetivos clave de la infraestructura energética y atacar no solo las patrullas del Ejército, sino también los vehículos civiles (McDermott, 2013).

2 Relativo a la protección de las víctimas de los conflictos armados sin carácter internacional (protocolo II), del 8 de junio de 1977, y del cual Colombia es signatario. 
estación genere una ventaja militar definida. Incluso, el Frente 61 emitió posteriormente un comunicado en el que no solo admite la responsabilidad del ataque, sino que además declara que la orden del cese al fuego no llegó a la unidad que realizó la acción, pero no se refiere a la afectación directa a la contraparte.

En tercer lugar, al emplear los elementos biológicos alrededor del bien civil, los miembros del Frente 61 desconocieron el principio de distinción de que trata el artículo 52(3) PA I, en cuanto este prevé que "en caso de duda acerca de si un bien que normalmente se dedica a fines civiles, tal como un lugar de culto, una casa u otra vivienda o una escuela, se utiliza para contribuir eficazmente a la acción militar, se presumirá que no se utiliza con tal fin”. Al respecto de ese artículo, el profesor Olásolo (2007) menciona que

solo cuando el atacante esté convencido de que están siendo utilizados por el enemigo para alojar a la tropa o para contribuir de otra manera a su acción militar, podrán ser objeto de ataque, para lo cual será normalmente necesario que se adopten las medidas de precaución previstas en el art. 57(2) PA I.

Esta precaución que establece el Protocolo Adicional para que un bien pueda ser considerado como objetivo militar está estrechamente relacionada con la ventaja militar que se espera obtener, siempre y cuando no suponga un hecho ilícito en el derecho humanitario, por ejemplo, "las muertes y las lesiones causadas a combatientes - o a no combatientes que participan directamente en las hostilidades_ que pertenecen a la parte adversa, $y$, por otra parte, la destrucción y el daño causado al equipamiento y las infraestructuras de la parte adversa” (Olásolo, 2007, p. 63).

En otras palabras, en el análisis del caso estudiado es fundamental definir si la ventaja militar que obtendría la guerrilla al considerar la estación de policía como un objetivo militar está directamente relacionada con el principio de proporcionalidad en el ataque (si en efecto se constata la existencia de un objetivo militar). No obstante, se debe recordar que ciertos bienes que normalmente son utilizados con fines civiles, en el marco de un conflicto armado también pueden ser empleados con propósitos militares, en virtud del alcance y objetivos políticos del conflicto armado de que se trate.

Como en el caso de estudio no parece que el bien esté claramente definido como objetivo militar, se estudiarán brevemente los elementos subjetivos y objetivos (contextuales y específicos) de la acción, con el ánimo de establecer si esta constituye una infracción al $\mathrm{DIH}$, específicamente, la comisión de un crimen de guerra: ataque a bienes civiles.

Respecto a los elementos subjetivos, vale la pena mencionar que el ataque de las FARC encaja en un patrón de conducta más amplio que tiene la organización en el territorio donde opera y que, en particular, existe una campańa de siembra de explosivos improvisados y minas antipersonales en contra de la infraestructura civil. El hecho de que el Frente 61 de las FARC haya usado gases tóxicos en el sur del departamento del Huila y después haya minado el área para evitar que fuera reparada, constituye una acción violatoria del DIH. El punto más crítico de estas acciones tuvo lugar en el año 2002, cuando 
la organización utilizó durante un corto tiempo armas químicas y biológicas, con lo cual constituyó dicha práctica en una política general (elemento contextual de carácter contextual jurisdiccional —condición objetiva de procedibilidad—).

Así pues, el ataque a la estación de policía de San Alfonso que realizaron los miembros del Frente 61 forma parte de una estrategia sistemática de afectación indiscriminada de la población civil. Es decir, esta acción responde a la comisión de actos individuales que siguen patrones predeterminados - cuya ocurrencia no sigue un patrón aleatorio-y que tuvieron como propósito presionar las opiniones políticas respecto a temas sensibles de la población civil de los cascos urbanos, afectar a la población civil y militar indiscriminadamente. Asimismo, cabe mencionar que estos atentados encarecen los costos de equipamiento que necesitan las unidades para atender estas problemáticas (elemento contextual de carácter material —relación entre la conducta típica y el conflicto armado—).

Finalmente, para concluir el análisis, se puede plantear que el uso de minas antipersonales (MAP) y artefactos explosivos improvisados (AEI), así como el uso de armas químicas, biológicas o nucleares en el marco del conflicto armado constituyen una infracción al DIH, específicamente, una tipificación objetiva como crimen de guerra, toda vez que genera sufrimientos innecesarios a los combatientes afectados y no distingue entre civiles y no civiles.

\section{Un manual para la atención de incidentes}

\section{Justificación}

La posibilidad de que organizaciones irregulares usen este tipo de materiales pone en riesgo la protección de los Derechos Humanos que debe garantizar el Estado colombiano, pues afectan a la población civil y no dan cuenta de la intención de desescalar la magnitud de la violencia.

Esta situación plantea la necesidad de que los organismos civiles y militares definan acciones concretas para gestionar adecuadamente los casos en que las organizaciones criminales usen este tipo de armamento, con el ánimo de reducir el riesgo y la vulnerabilidad de la población civil y de las instituciones castrenses. Parte importante de estas acciones es reconocer el marco normativo y operativo existente para tratar este tipo de amenazas, particularmente el artículo 81 de la Constitución de 1991, que prohíbe la fabricación, importación, posesión y uso de armas químicas, biológicas y nucleares, así como la introducción al territorio colombiano de residuos nucleares y desechos tóxicos (Ministerio de Ambiente, Vivienda y Desarrollo Territorial \& Consejo Colombiano de Seguridad, 2003). Es decir que queda expresamente prohibido para las instituciones públicas o los ciudadanos la posesión de armas de destrucción masiva (AMD). Sin embargo, como se ha podido observar en diversos medios de comunicación y en distintos procesos judiciales, las FARC han traficado con uranio enriquecido y, eventualmente, con otras armas quí- 
micas (IISS, 2011), lo cual genera un riesgo estratégico (Torrijos, 2009) que obliga a las Fuerzas Militares a estar preparadas para enfrentar este tipo de amenazas.

Actualmente, entre las armas de destrucción masiva que se pueden encontrar están las nucleares, biológicas y químicas. Ahora bien, si se tiene en cuenta que un conflicto asimétrico como el colombiano genera un alto flujo de recursos (Carbó, 2001) y que este posibilita la obtención de este tipo de armamento por parte de los grupos ilegales, resulta imprescindible que los organismos de seguridad se preparen para dimensionar y contrarrestar las amenazas derivadas de su uso en el contexto local y en el escenario estratégico (Duncan, 2007). Sobre todo, considerando que las FARC plantean la combinación de todas las formas de lucha para tomarse el poder (Shifter, 1999), lo cual ha impactado sus posibilidades de crecimiento (Echandía, 1997).

Un insumo clave de esta preparación es la Convención sobre las Armas Químicas (CAQ), que ha estado vigente durante un decenio. Particularmente, la comunidad internacional se sirve de este instrumento para eliminar, definitivamente, toda posibilidad de desarrollar, producir, emplear, almacenar o transferir estas armas atroces. Su función principal consiste en aplicar las disposiciones de la Convención sobre las Armas Químicas, a fin de hacer realidad la aspiración de la Organización para la Prohibición de Armas Químicas (OPAQ), es decir, un mundo libre de armas químicas donde se promueva la cooperación para el uso pacífico de la química. Con esto se pretende contribuir, de forma decisiva, a la seguridad y estabilidad internacionales, al desarme general y absoluto, y al desarrollo económico mundial.

La Convención entró en vigor para Colombia el 4 de junio de 2000, mediante la aprobación de la Ley 525 de 1999 del Congreso de la República. El 10 de julio de 2002 fue aprobado el Decreto 1419, mediante el cual se creó en Colombia la Autoridad Nacional para la Prohibición de las Armas Químicas (Anproaq). La Autoridad está conformada por los ministerios de Relaciones Exteriores (Presidencia), Defensa Nacional (Secretaría Técnica Indumil), Agricultura y Desarrollo Rural, Protección Social, Ministerio de Comercio, Industria y Turismo, Ministerio de Ambiente, Vivienda y Desarrollo Territorial.

Una forma eficiente de preparar a las instituciones para contener y/o gestionar este tipo de amenazas es diseñar e implementar un manual de amenazas nucleares, biológicas y químicas, con el fin de dar a conocer las normas, procedimientos y métodos más adecuados que se pueden utilizar en la atención e identificación de incidentes y accidentes con estos materiales peligrosos. Esta estrategia, tendiente a concentrar las acciones socio-institucionales en contra de los fenómenos criminales, tiene antecedentes en políticas gubernamentales que han pretendido ser vinculantes en el ordenamiento jurídico colombiano (Leal, 2006).

En todo caso, estrategias de este tipo permiten condensar un conjunto de recomendaciones, a manera de manual, para que las instituciones ofrezcan una atención adecuada y especializada en cada uno de los casos que puedan presentarse en cualquier parte del territorio nacional. Dicho manual no solo permitiría coordinar y armonizar las funciones 
de las instituciones, sino que además permitiría la coordinación entre los diferentes cuerpos de seguridad y defensa en materia de competencias y jurisdicciones (Dupuy, 2005).

Cabe seńalar que solo mediante la coordinación de las instituciones gubernamentales y las Fuerzas Militares, a través de procedimientos y procesos claros y condensados, es posible garantizar el cumplimiento de la Ley 525 de 1999 y de las disposiciones establecidas por el Decreto 1419. Si se coordinan las instituciones pertinentes, será posible reducir el costo asociado a las labores de verificación sistemática mediante inspección in situ y vigilancia con instrumentos in situ, el transporte de material peligroso (Decreto 1609 de 2002), así como las demás actividades que verifican la existencia y control de sustancias nucleares, biológicas y químicas.

Asimismo, un manual militar que regule la materia les facilitaría a los equipos especializados reconocer los agentes químicos, bacteriológicos o nucleares, a través de los cuales se limitaría los riesgos derivados de esta amenaza. En este sentido, el manual tiene como objeto dar a conocer las normas, procedimientos y métodos más adecuados que puede utilizar el Ejército Nacional para atender e identificar incidentes y accidentes con sustancias peligrosas.

Otro aspecto de esta propuesta es que se facilita el conocimiento y la descripción de los equipos de protección y de descontaminación por ataque con sustancias peligrosas, al tiempo que propicia el conocimiento de las sustancias químicas que, siendo comerciales, se pueden convertir en agentes tóxicos y, por ende, tienen la potencialidad de ser letales para la vida humana. Adicionalmente, se propone reconocer la organización del Sistema de Comando de Incidentes (SCI).

\section{El manual}

La identificación, atención de incidentes y descontaminación de áreas afectadas por el uso de sustancias nucleares, biológicas y químicas (NBQ), es un tema que no está claramente incorporado en los planes de gestión de riesgo de entidades territoriales, y tampoco presenta un procedimiento de atención definida en los procedimientos atendidos por las Fuerzas Militares, por lo cual es importante definir un protocolo, teniendo como base la normatividad asociada, las capacidades y los roles de las instituciones públicas.

En un contexto de amenazas irregulares a la seguridad de las personas y/o el medio ambiente, asociado a déficits en el control de la comercialización o el uso de sustancias NBQ que representan por su naturaleza un riesgo para la salud humana o el medio ambiente, las instituciones públicas están llamadas a identificar los escenarios de riesgo en la ocurrencia de eventos e incidentes y, en particular, en los procedimientos de descontaminación de áreas afectadas en incidentes con sustancias NBQ. En entornos locales del país, en los cuales las deficiencias del Estado son visibles, la atención oportuna de incidentes con estas sustancias, así como una efectiva y sostenible descontaminación de las áreas afectadas, puede promover el fortalecimiento institucional local y, por lo tanto, garantizar 
la protección de derechos fundamentales y la atención de responsabilidades públicas derivadas del ordenamiento jurídico nacional.

En este sentido, es necesario precisar que para garantizar la descontaminación de áreas afectadas con sustancias NBQ a través de unidades del Ejército Nacional, se requiere plantear un esquema de atención que defina el proceso de valoración de la amenaza, la planificación de la intervención, la ejecución y la evaluación de esta. Con este objetivo presente, el manual debe contemplar el que las unidades realicen la identificación de escenarios de amenazas NBQ y la descripción de capacidades y estrategias a través de las cuales las unidades capacitadas para la descontaminación de áreas afectadas pueden desarrollar las actividades de descontaminación. Para tal fin, se deben analizar algunos requisitos generales para la descontaminación de áreas afectadas por incidentes NBQ.

En relación con estos contextos, resulta necesario avanzar en la caracterización y tipificación de dichos escenarios de afectación o "escenarios peligros", así como de las estrategias de intervención, particularmente la descontaminación de áreas afectadas, en los cuales las Fuerzas Militares pueden desenvolverse. Al respecto, Píta (2005) enfatiza en que se debe avanzar en

[e]l equipo y los procedimientos necesarios para detectar e identificar agentes químicos o biológicos de guerra, [los cuales] deben estar preparados y ensayados previamente en los diferentes niveles de la amenaza, con el fin de que esa detección o identificación se lleve a cabo lo antes posible y las alertas y/o alarmas sean dadas de forma oportuna. Esto permitirá, también, adoptar las medidas de protección adecuadas del personal que pueda verse expuesto.

Pero una vez planteados los escenarios ideales, las unidades militares destinadas para atender este tipo de incidentes deben prever cuidadosamente las operaciones de descontaminación necesarias para garantizar una atención eficiente y efectiva. El primer paso en el diseño de estas operaciones consiste en tener una comunicación previa con productores, comercializadores, transportadores y usuarios, con el fin de conocer las características generales y específicas de los productos y sus condiciones de manipulación. Dicha información es vital en cualquier escenario de limpieza de áreas contaminadas y, particularmente, en zonas frágiles y de abundante biodiversidad (variedad de especies animales y vegetales), como el piedemonte de la cordillera Oriental, la cuenca del río Magdalena, entre otras del territorio colombiano.

Asimismo, se debe establecer por escrito los procedimientos que se realizarán para actuar con seguridad frente a un posible incidente. Los lineamientos que definen los procedimientos son: $i$ ) identificar el producto y evaluar el incidente, ii) establecer comunicación con el Sistema de Comando de Incidentes (SCI), iii) delimitar y cerrar el área, iv) contener la amenaza, $v$ ) limpiar el personal y los equipos, y vi) descontaminar el área (tabla 1). 
Tabla 1. Lineamientos para la atención de amenazas NBQ

\begin{tabular}{|c|c|}
\hline Lineamiento & Descripción \\
\hline Identifique el producto & $\begin{array}{l}\text { - Evalúe el área. } \\
\text { - Localice el origen y naturaleza del incidente. } \\
\text { - Establezca las características, mecanismos de expansión y riesgos del } \\
\text { producto. } \\
\text { - Identifique los riesgos y magnitudes de impacto. } \\
\text { - Lleve una minuta de los hechos. } \\
\text { - Realice las acciones iniciales de contención teniendo dos prioridades: } \\
\text { el origen del evento o la contención de la zona que pueda tener un } \\
\text { mayor efecto. } \\
\text { - Evite el contacto directo con la sustancia. }\end{array}$ \\
\hline Comuníquese con el SCI & $\begin{array}{l}\text { - Entregue la información que pueda a la supervisión directa, para que se } \\
\text { proceda al control de la emergencia. Esto incluye equipos, materiales y } \\
\text { áreas afectadas; señalando ubicación, productos comprometidos, canti- } \\
\text { dad, su dirección y condición actual. } \\
\text { - Busque más información. } \\
\text { - Recurra a asesoría externa, si es necesaria. } \\
\text { - Esté presto a mantener comunicaciones. }\end{array}$ \\
\hline Delimite y cierre el área & $\begin{array}{l}\text { - Difunda alertas sobre el incidente para evitar pérdidas civiles o militares. } \\
\text { - Establezca medidas de circulación. } \\
\text { - Acordone el lugar rodeando el área contaminada. } \\
\text { - Rodee con materiales absorbentes los equipos o materiales. } \\
\text { - Apague todo equipo o fuente de ignición. } \\
\text { - Disponga de algún medio de control en caso de ignición. }\end{array}$ \\
\hline Contenga la amenaza & $\begin{array}{l}\text { - Identifique y disponga los elementos de protección personal necesarios. } \\
\text { - Localice el origen del incidente y las medidas de control en dicho nivel. } \\
\text { - Tome medidas de mitigación si la contaminación es muy amplia. } \\
\text { - Evite contaminar el medio ambiente. }\end{array}$ \\
\hline $\begin{array}{l}\text { Limpie al personal } \\
\text { y los equipos }\end{array}$ & $\begin{array}{l}\text { - Intente recuperar el producto. } \\
\text { - Absorba o neutralice. Para el caso de ácidos o bases, proceda a neutralizarlos. } \\
\text { - Lave la zona contaminada con agua, en caso de que no exista contrain- } \\
\text { dicación. } \\
\text { - Seńalice los recipientes donde se van depositando los residuos. Todos los } \\
\text { productos recogidos deben tratarse como residuos peligrosos. }\end{array}$ \\
\hline Descontamine el área & $\begin{array}{l}\text { - Disponga de una zona de descontaminación. } \\
\text { - Lave los equipos y la ropa utilizada. } \\
\text { - Las personas que intervinieron en la descontaminación deben bañarse. }\end{array}$ \\
\hline
\end{tabular}

Fuente: elaboración propia. 
Las operaciones de descontaminación en incidentes con sustancias NBQ hacen necesario que se conforme un Sistema de Comando de Incidentes (SCI), en el cual se combinan instalaciones, equipamiento, personal, protocolos, procedimientos y comunicaciones. Este debe operar en una estructura organizacional común, con la responsabilidad de administrar los recursos asignados para cumplir efectivamente los objetivos pertinentes a un evento, incidente u operación. Este sistema de comando, que puede ser dirigido por un comando unificado, tiene como objetivo asegurar el despliegue rápido, coordinado y efectivo de los recursos y minimizar la alteración de las políticas y procedimientos operativos propios de cada una de las instituciones que responden a la atención de un evento con sustancias NBQ.

Un esquema logístico y de comunicación adaptado a todo el territorio nacional debe prever la coordinación con la autoridad local de gestión del riesgo, con el fin de identificar (o, ante su ausencia, formar) una estructura organizacional que esté establecida y funcionando, en la cual se defina el régimen de responsabilidades y procedimientos que garanticen la identificación de las necesidades de entrenamiento y los requerimientos y evaluación de la competencia laboral de los sujetos llamados a atender los incidentes con sustancias NBQ.

Para un desarrollo eficiente de este tipo de operaciones se debe contar con una sección de atención, la cual es responsable de proveer instalaciones, servicios y materiales, incluyendo el personal que opera los equipamientos solicitados para atender el incidente. Esta sección es indispensable cuando las operaciones se cumplen en grandes extensiones y cuando son de larga duración. Las funciones de la sección son apoyar exclusivamente a quienes atienden el incidente. Por ejemplo, incluye una unidad médica que proporciona atención al personal de respuesta al incidente y no a las víctimas.

\section{Conclusión}

Un manual de sustancias NBQ pretende minimizar las muertes y/o contaminaciones por la acción de agentes químicos, tanto de personal militar como de la población civil que pueda ser víctima de acciones terroristas. Con ello, el Ejército Nacional contribuye al conocimiento de las normas, procedimientos y métodos más adecuados que se pueden emplear en la atención e identificación de incidentes y accidentes con estas sustancias. Con esto brindará, además, una atención adecuada y especializada en cada uno de los casos que puedan presentarse en cualquier parte del territorio nacional.

\section{Agradecimientos}

El autor agradece a la Escuela de Ingenieros Militares del Ejército Nacional de Colombia.

\section{Declaración de divulgación}

El autor declara que no existe ningún potencial conflicto de interés relacionado con el artículo. La presente investigación forma parte de un proyecto de investigación en la línea Ingeniería Civil y Militar Aplicada a la Gestión del Conocimiento, desarrollado en 
el marco de la Maestría en Gestión del Riesgo de la Escuela de Ingenieros Militares del Ejército Nacional de Colombia.

\section{Financiamiento}

El autor no declara fuente de financiamiento para la realización del artículo.

\section{Sobre los autores}

John Aldrin Hernández Méndez es oficial en actividad del Ejército Nacional de Colombia con el grado de Coronel. Profesional en Ciencias Militares.

\section{Referencias}

Carbó, E. (2011). ¿Guerra civil? El lenguaje del conflicto en Colombia. Bogotá, D.C.: Cambio.

Duncan, G. (2007). Los señores de la guerra: de paramilitares, mafiosos y autodefensas en Colombia. Bogotá, D. C.: Planeta.

Dupuy, P. (2005). Reformas y contrarreformas en la Policía colombiana. Bogotá, D. C.: Fundación Seguridad y Democracia.

Echandía, C. (1997). Expansión territorial de la guerrilla colombiana: geografía, economía y violencia [Documento de trabajo del Programa de Estudios sobre Seguridad, Justicia y Violencia N.o 1]. Recuperado de https://www.academia.edu/30078985/Expansi\%C3\%B3n_Territorial_de_la_Guerrilla_ Colombiana_Geograf\%C3\%ADa_Econom\%C3\%ADa_y_Violencia

Echandía, C. (2011). Situación actual de las FARC: un análisis de los cambios en las estrategias y la territorialidad (1990-2011). Informes FIP, 13, 25-31.

IISS. (2011). The Farc Files: Venezuela, Ecuador and the secret archive of 'Raúl Reyes' [documento en línea]. Recuperado de https://www.iiss.org/en/publications/strategic\%20dossiers/issues/the-farc-files--venezuela--ecuador-and-the-secret-archive-of---39-ra--250-1-reyes--39-8716

Las FARC y el ELN usan armas químicas. (29 de marzo de 2003). El País. Recuperado de http://historico. elpais.com.co/paisonline/notas/Marzo292003/nal5.html

Leal Buitrago, F. (2006). En la encrucijada, Colombia siglo XXI. Bogotá, D. C.: Norma.

McDermott, J. (20 de mayo de 2013). Actividades criminales de las FARC y las ganancias de la guerrilla [documento en página web]. Insight Crime. Recuperado de http://es.insightcrime.org/investigaciones/actividades-criminales-farc-y-ganancias-de-la-guerrilla

Ministerio de Ambiente, Vivienda y Desarrollo Territorial \& Consejo Colombiano de Seguridad. (2003). Guias para manejo seguro y gestión ambiental de 25 sustancias quimicas [documento en línea]. Recuperado de http://www.minambiente.gov.co/images/AsuntosambientalesySectorialyUrbana/ pdf/sustancias_qu\%C3\%ADmicas_y_residuos_peligrosos/guia_25_sustancias.pdf

Olásolo Alonso, H. (2007). Ataques contra personas y bienes civiles y ataques desproporcionados. Valencia, España: Tirant.

Píta, R. (2005). Componentes de la defensa química y biológica en operaciones militares. España: Real Academia Nacional de Farmacia.

Shifter, M. (1999). Colombia on the brink: there goes the neighborhood. Foreign Affairs, 74, 14-20.

Torrijos, V. (2009). Los argumentos para alejarse de la paz en Colombia: ideas para alejarse de la guerra. En V. Torrijos, Asuntos estratégicos, seguridad y defensa (pp. 59-65). Bogotá: Universidad del Rosario. 